\title{
Active Materials based on Implanted Si for Obtaining Intermediate Band Solar Cells
}

\author{
Kefrén Sánchez , Irene Aguilera , Pablo Palacios and Perla Wahnón
}

Keywords: Intermediate band, photovoltaic materials, first-principles calculations, Si

\begin{abstract}
First-principles calculations carried out for compounds based on Si implanted with different species, as $\mathrm{Ti}$ or chalcogens $(\mathrm{S}, \mathrm{Se}, \mathrm{Te})$, show them as solid candidates to intermediate band (IB) photovoltaic materials. This DFT study predicts electronic structures, formation energies, relaxed atomic structures, optoelectronic properties, diffusion paths, for supercells containing up to several hundreds of atoms. The knowledge of Si-based devices is a relevant factor to facilitate the creation of an IB solar cell. Crystalline samples with a concentration of Ti several orders of magnitude above the solubility limit have been already grown. Formation energy calculations agree with the experiment in showing mainly interstitial implantation. Calculated electronic structure presents an IB, which is in agreement with electrical measurements and models, and is expected to cause an increase of the absorption coefficient across the solar spectrum. Chalcogen-implanted Si is an efficient IR absorber when implantation is carried out at ultra-high concentrations. Substitutional implantation produces a filled band inside Si band-gap and our calculations predict that plausible co-doping with IIIA atoms (as Al, B) would allow to obtain an IB fulfilling all the needed requirements.
\end{abstract}

\section{Introduction}

Nowadays silicon is the best known semiconductor and the most broadly used material for photovoltaic applications. For this reason, a mature technology is available for developing devices based on it.

The quest for intermediate-band materials has advanced fast during the recent years [1-7]. Growth of experimental samples has been added to the prediction of several compounds. Indeed different implementations of intermediate-band solar cells have been proposed. The main attractive of this kind of solar cells consists on the predicted limit of its thermodynamic efficiency [8], being much higher than the corresponding to conventional solar cells.

However, the duty of obtaining an intermediate-band solar cell with enhanced efficiency is ongoing. Construction of this device from an active material based on silicon seems to be an interesting short cut in the path to showing clearly its operating principles.

As a result, different proposals of intermediate-band materials based on $\mathrm{Si}$ have appeared recently. On the one hand, Ti-implanted $\mathrm{Si}$ is expected to form an intermediate band when Ti atoms occupy interstitial sites [9]. Crystalline samples of this material up to Ti concentrations around $10^{20}$ $\mathrm{cm}^{-3}$ have been already grown and characterized [10-13].

On the other hand, substitution of Si atoms by chalcogens ( $\mathrm{S}, \mathrm{Se}, \mathrm{Te})$ shows an intermediate band when additional p-doping at similar concentration is carried out [14]. Although coimplantation of this compound has not been carried out yet, samples with a chalcogen concentration close to the $0.5 \%$, which was analyzed theoretically, have been obtained $[15,16]$. Therefore the present background in Si doping allows expecting that these samples could be co-implanted at similar concentrations. 
Additionally, the abovementioned compounds share a common synthesis method. It consists on combining ion implantation with pulsed laser melting (PLM). This technique allows achieving implantations with concentrations much above the solubility limit.

In order to compare the features of these materials an $a b$ initio study is carried out in this contribution. The method in which the work is based is explained in the next section. After that, results obtained will be analyzed. Finally, a summary of the study and the conclusions found will be detailed in the last section.

\section{Method}

First-principles calculations presented here belong to the framework given by the Density Functional Theory (DFT) [17,18] at the Generalized Gradient Approximation (GGA) level using the PW91 functional [19]. Plane-wave based VASP program [20,21] was used and the inert core electrons were taken into account through Projector Augmented Wave (PAW) potentials[22]. The sampling of the Brillouin zone was carried out using the method of Monkhorst and Pack with a $\Gamma$ centered $4 \times 4 \times 4$ grid.

The structures considered here are derived from a $\mathrm{Si}_{216}$ cell. This supercell was constructed in turn from the conventional $\mathrm{Si}_{8}$ unit cell of bulk silicon. All of the atomic structures were relaxed to forces below $0.01 \mathrm{eV} / \AA$ and stresses in the lattice parameters were reduced down to $0.1 \mathrm{GPa}$.

All of the optoelectronic properties analyzed in the text are obtained from the dielectric function. The latter is calculated by carrying out a sum over independent direct transitions between KohnSham eigenvalues [23]. Convergence of the optical properties has been ensured using an $8 \times 8 \times 8$ Monkhorst-Pack grid of the reciprocal lattice and including 800 electronic levels for each k-point. Calculations were carried out by means of the OPTICS program [24].

\section{Results}

Formation energies. A summary of predicted formation energies is presented in Table 1. In general terms, $\mathrm{Ti}_{i}$ implantation (where $\mathrm{Ti}_{i}$ denotes interstitial $\mathrm{Ti}$ ) is more unfavorable than most of co-implantation containing chalcogens. Nevertheless it must be noted that this does not mean necessarily that $\mathrm{Ti}_{i} \mathrm{Si}_{216}$ material be more unlikely. Indeed, experimental samples at concentrations close to $0.5 \%$, i.e. $2 \times 10^{20} \mathrm{~cm}^{-3}$, are already available. Furthermore, other implantations with similar formation energies have been also carried out by means of non-equilibrium techniques.

Table 1. Summary of formation energies and structural properties of intermediate-band materials based on implanted Si. Lattice parameter calculated for bulk Si is $5.464 \AA$.

\begin{tabular}{|c|c|c|c|}
\hline Compound & $\begin{array}{c}\text { Formation energy } \\
{[\mathrm{eV}]}\end{array}$ & $\begin{array}{c}\text { Relaxed lattice } \\
\text { parameter }[\AA]\end{array}$ & $\begin{array}{c}\text { Difference regarding Si } \\
\text { lattice parameter }[\AA]\end{array}$ \\
\hline $\mathrm{Ti}_{i} \mathrm{Si}_{216}$ & +1.51 & 5.466 & +0.002 \\
\hline Remote $\mathrm{B}_{S i} \mathrm{~S}_{S i} \mathrm{Si}_{214}$ & -0.49 & 5.458 & -0.006 \\
\hline Near $\mathrm{B}_{S i} \mathrm{~S}_{S i} \mathrm{Si}_{214}$ & -0.64 & 5.460 & -0.004 \\
\hline Remote $\mathrm{Al}_{S i} \mathrm{~S}_{S i} \mathrm{Si}_{214}$ & +0.23 & 5.467 & +0.003 \\
\hline Near $\mathrm{Al}_{S i} \mathrm{~S}_{S i} \mathrm{Si}_{214}$ & -0.72 & 5.466 & +0.002 \\
\hline Remote $\mathrm{B}_{S i} \mathrm{Se}_{S i} \mathrm{Si}_{214}$ & -0.45 & 5.460 & -0.004 \\
\hline Near $\mathrm{B}_{S i} \mathrm{Se}_{S i} \mathrm{Si}_{214}$ & -0.62 & 5.461 & -0.003 \\
\hline Remote $\mathrm{Al}_{S i} \mathrm{Se}_{S i} \mathrm{Si}_{214}$ & +1.64 & 5.469 & +0.005 \\
\hline Near $\mathrm{Al}_{S i} \mathrm{Se}_{S i} \mathrm{Si}_{214}$ & +0.81 & 5.468 & +0.004 \\
\hline Remote $\mathrm{B}_{S i} \mathrm{Te}_{S i} \mathrm{Si}_{214}$ & +0.15 & 5.463 & -0.001 \\
\hline Near $\mathrm{B}_{S i} \mathrm{Se}_{S i} \mathrm{Si}_{214}$ & -0.24 & 5.463 & -0.001 \\
\hline Remote $\mathrm{Al}_{S i} \mathrm{Te}_{S i} \mathrm{Si}_{214}$ & +2.22 & 5.472 & +0.008 \\
\hline Near $\mathrm{Al}_{S i} \mathrm{Te}_{S i} \mathrm{Si}_{214}$ & +1.48 & 5.471 & +0.007 \\
\hline
\end{tabular}


In principle other configurations involving interstitial location of one or both the introduced species could compete with the double-substitutional co-implantation of Si. However, it is wellknown that all of these species mostly substitute Si atoms when they are isolated impurities as the substitution is certainly the most stable implantation process. In addition, the study of the complete range of configurations including interstitial implantations would require the analysis of a great amount of complex combinations, although it is noticed that such study can be interesting as future work.

Observing chalcogen-implanted $\mathrm{Si}$ exclusively, $\mathrm{B}$ containing compounds present lower formation energies than $\mathrm{Al}$. This may be due to the fact that $\mathrm{B}$ radius is more similar to $\mathrm{Si}$ than $\mathrm{Al}$ is, being thus adapted better to the Si lattice. Additionally B is smaller than Si while Al is bigger. As a result, stress produced by chalcogens introduction, which are in general also bigger, is partially compensated only by B.

Structural properties. The relaxation of the atomic structure of $\mathrm{Ti}_{i} \mathrm{Si}_{216}$ compound gives place to a lattice parameter of $5.466 \AA$, which is reasonably close to the $5.464 \AA$ calculated for bulk Si at GGA level. Therefore, it is expected that this material do not present noticeable strains if the concentration of Ti remains at moderate values.

Conversely, lattice parameter of co-implanted materials depends on the specific combination of chalcogen atom and group III element, while the distance between both substituting atoms does not seem to be a main factor for the size of the resulting unit cell. Only $\mathrm{Al}_{S i} \mathrm{Te}_{S i} \mathrm{Si}_{214}$, and maybe $\mathrm{B}_{S i} \mathrm{~S}_{S i} \mathrm{Si}_{214}$, could cause some appreciable strain in the unit cell regarding to bulk $\mathrm{Si}$.

Electronic properties. The origin of the intermediate band in the two kinds of intermediateband materials based on Si differs completely.

On the one hand, the interstitial implantation of the introduced Ti atom results into a unusual splitting in the Ti $3 d$ electronic states into low energy $t_{2 g}$ states and high-energy $e_{g}$ states in spite of initially the nearest neighbors are placed in tetrahedral coordination (see [9]). The latter get energies in the range of the conduction band whereas the former are placed in the central region of the gap of the host semiconductor. As a result two narrow bands appear isolated within the gap, being the majority-spin one fully occupied with three electrons and the minority-spin one partially occupied (1/3). The latter therefore complies with all the intermediate-band requirements.

On the other hand, replacement of $\mathrm{Si}$ is carried out by $\mathrm{S}$, Se or Te atoms gives place to qualitatively similar electronic structures independently from the chalcogen species [14]. In all of the cases a full band appears within the gap of Si. This band is occupied by one electron per spin channel. The co-implantation with group III elements $(\mathrm{B}, \mathrm{Al})$ at comparable concentrations allows obtaining a partial occupation without modifying the band energies considerably. These annotations are obtained for all possible combinations of chalcogen and group III species and are independent from variation in the distance between both substituting atoms. Consequently, intermediate bands are observed in all of these cases and therefore an assortment of intermediate-band materials based on $\mathrm{Si}$ is provided.

Optical properties. Optical absorption coefficients of the compounds described in the previous subsection are compared in Fig. 1. For the sake of clarity, only those chalcogen-containing compounds co-implanted with boron have been included given that those co-implanted with Al produce almost identical absorption features [14].

All of the intermediate-band materials show absorption in the range of photon energies below the direct gap. In the case of Ti-implanted Si the absorption does not vary significantly across that region whereas in the compounds containing chalcogens, especially in the case of those with Te substitution, a main peak observed at energies below $1 \mathrm{eV}$ is followed by a tail appearing at higher energies. 


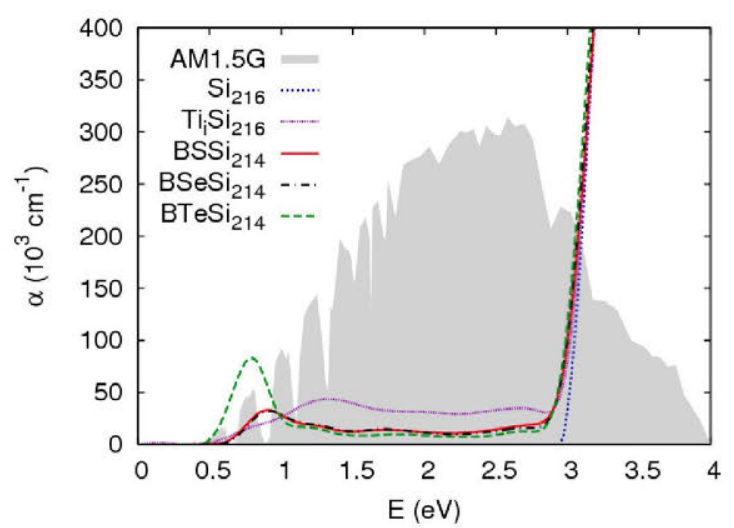

Figure 1. Optical absorption coefficients obtained for several intermediate-band materials based on $\mathrm{Si}$. Optical absorption of bulk $\mathrm{Si}$ is also included and the solar spectrum is represented as a reference.

Homogeneous co-implanted compounds have been analyzed in Fig. 1. However, chalcogenimplanted Si results into lower ground-state energies when the co-implantation with group III atoms is carried out at close sites. Therefore, the optoelectronic features calculated for closely coimplanted $X_{S i} \mathrm{~S}_{S i} \mathrm{Si}_{214}, X_{S i} \mathrm{Se}_{S i} \mathrm{Si}_{214}$, and $X_{S i} \mathrm{Te}_{S i} \mathrm{Si}_{214}(X=\mathrm{B}, \mathrm{Al})$ are represented in Fig. 2, Fig. 3, and Fig. 4, respectively.

In Fig. 2 it can be seen that $\mathrm{Si}$ co-implanted with close $\mathrm{S}$ and $X$ substituting atoms results in a compound that again presents optical absorption below Si band gap, which was expected in view of the band structures obtained elsewhere [14]. Looking at detail the absorption coefficients of Fig. 2 (c), a decrease is observed when comparing the results with the corresponding to $\mathrm{S}_{\mathrm{Si}} \mathrm{Si}_{215}$, which is mainly due to a reduction in the transitions from the intermediate to the conduction band. Although a similar effect had been observed for the remote configuration of the atoms [14], in the coimplantations presented in Fig. 2 the decrease seems to be less pronounced.
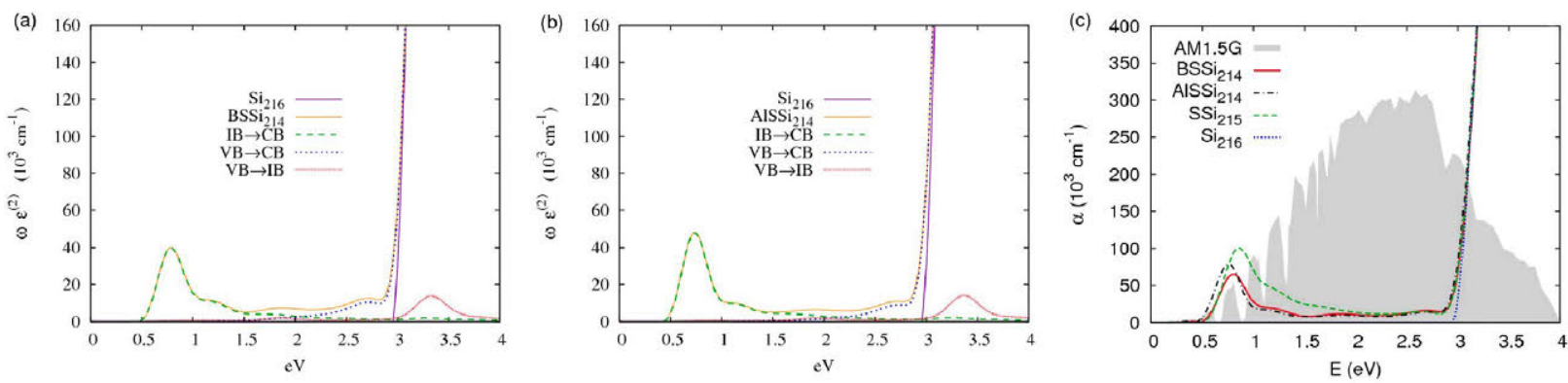

Figure 2. Imaginary part of the dielectric function calculated for (a) $\mathrm{B}_{S i} \mathrm{~S}_{S i} \mathrm{Si}_{214}$ and (b) $\mathrm{Al}_{S i} \mathrm{~S}_{S i} \mathrm{Si}_{214}$ compounds. Both total and partial contributions have been included. Also the optical-absorption coefficient is represented for these compounds (c) and compared with the obtained for $\mathrm{S}_{S i} \mathrm{Si}_{215}$ and bulk Si.

Analogous considerations can be established from the co-implanted compounds containing Se. Although the optical absorption for the close co-implantation shows values lower than $\mathrm{Si}$ exclusively implanted with Se, the reduction is smaller than the observed for the remote coimplantation. Additionally, a certain distinction between both group III co-implantations can be drawn in this case given that the $\mathrm{Al}_{\mathrm{Si}} \mathrm{Se}_{\mathrm{Si}} \mathrm{Si}_{214}$ compound seems to provide slightly enhanced absorption when compared with $\mathrm{B}_{\mathrm{Si}} \mathrm{Se}_{\mathrm{Si}} \mathrm{Si}_{214}$.

The concurrent co-implantation of close Te and group III elements produces again a reduction in the optical absorption for energies below the band gap, whose origin is inherent to the decrease observed in the transitions from the intermediate band to the conduction band (see Fig. 4). 

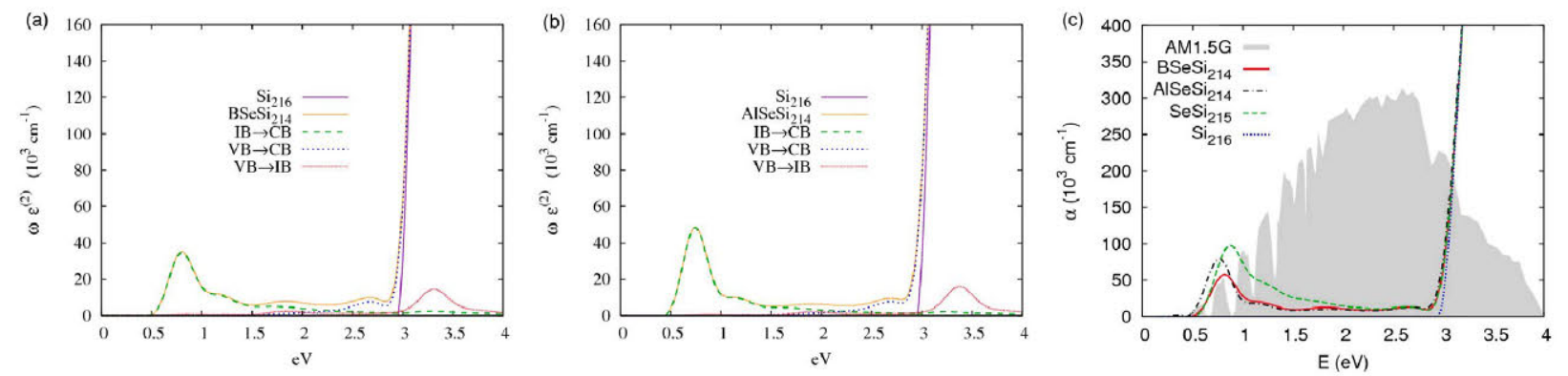

Figure 3. Imaginary part of the dielectric function calculated for (a) $\mathrm{B}_{S i} \mathrm{Se}_{S i} \mathrm{Si}_{214}$ and (b) $\mathrm{Al}_{S i} \mathrm{Se}_{S i} \mathrm{Si}_{214}$ compounds. Both total and partial contributions have been included. Optical-absorption coefficient is represented for these compounds (c) and compared with the obtained for $\mathrm{Se}_{S i} \mathrm{Si}_{215}$ and bulk Si .

Conversely, the enhancement obtained for the electronic excitations from the valence band to the intermediate band only appears for incoming photons with energies above $3 \mathrm{eV}$.
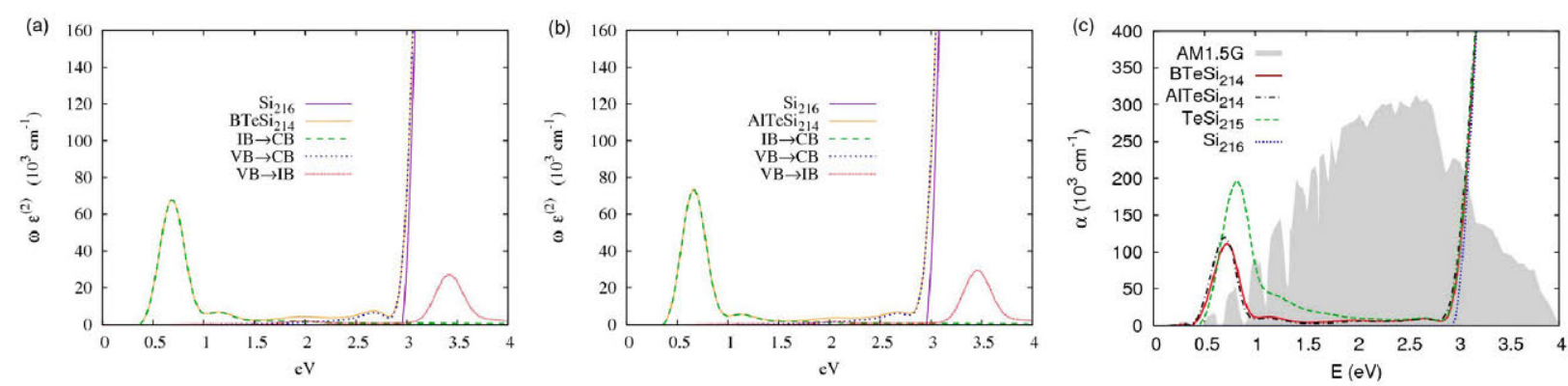

Figure 4. Imaginary part of the dielectric function calculated for (a) $\mathrm{B}_{S i} \mathrm{Te}_{S i} \mathrm{Si}_{214}$ and (b) $\mathrm{Al}_{S i} \mathrm{Te}_{S i} \mathrm{Si}_{214}$ compounds. Both total and partial contributions have been included. Also the optical-absorption coefficient is represented for these compounds (c) and compared with the obtained for $\mathrm{Te}_{S i} \mathrm{Si}_{215}$ and bulk Si.

Although substantial drop observed upon the introduction of the group III atom closely implanted $X_{S i} \mathrm{Te}_{S i} \mathrm{Si}_{214}$ compound presents appreciable absorption, mainly in the region around 0.7 $\mathrm{eV}$.

\section{Summary}

Two kinds of intermediate-band materials based on Si have been compared in the present theoretical work. The formation process of both compounds combines ion implantation with pulsed laser melting. The main advantage of constructing intermediate-band materials from bulk $\mathrm{Si}$ consists on the fact that the device technology based on this semiconductor reached already its mature stage.

The material containing $\mathrm{Ti}$ is characterized by the formation of an intermediate band from the $3 \mathrm{~d}$ electrons of the transition metal when the latter is placed at a tetrahedral interstitial, which is the most stable implantation way. Compounds that contain substituting chalcogens need to be p-doped to reduce the occupation of the isolated band and comply thus with all of the intermediate-bandmaterial requirements.

Examination of the optical properties shows optical absorption below the band gap with a similar magnitude for all of the candidates to intermediate-band material. Looking at detail, co-doped compounds based on chalcogen-implanted Si have slightly enhanced absorption properties when compared with the homogeneously co-implanted materials.

In conclusion, both kinds of implanted materials based on $\mathrm{Si}$ analyzed here seem feasible and can contribute to show the working principles of the intermediate band in a near future. 


\section{Acknowledgement}

The authors would like to acknowledge funding from GENESIS-FV (CSD2006-0004) and FOTOMAT (MAT2009-14625-C03-01) projects of the Spanish Ministerio de Ciencia e Innovación, as well as financial support from the Comunidad de Madrid through NUMANCIA-2 (S2009/ENE1477) project and the grant of K.S. Computer resources and assistance provided by the Centro de Supercomputación y Visualización de Madrid (CeSViMa) are also acknowledged.

\section{References}

[1] P. Wahnón, C. Tablero, Phys. Rev. B 65, 165115 (2002).

[2] P. Palacios, J. J. Fernández, K. Sánchez, J. C. Conesa, and P. Wahnón, Phys. Rev. B 73, 085206 (2006).

[3] P. Palacios, K. Sánchez, J. C. Conesa, P. Wahnón, Phys. Stat. Solidi (a) 203, 1395 (2006).

[4] P. Palacios, K. Sánchez, J. C. Conesa, J. J. Fernández, P. Wahnón, Thin Solid Films 515, 6280 (2007).

[5] R. Lucena, I. Aguilera, P. Palacios, P. Wahnón, J. C. Conesa, Chem. Maters. 20, 512 (2008).

[6] P. Palacios, I. Aguilera, K. Sánchez, J. C. Conesa, P. Wahnón, Phys. Rev. Lett. 101, 046403 (2008).

[7] I. Aguilera, P. Palacios, K. Sánchez, P. Wahnón, Phys. Rev. B 81, 075206 (2010).

[8] A. Luque, A. Martí, Phys. Rev. Lett. 78, 5014 (1997).

[9] K. Sánchez, I. Aguilera, P. Palacios, P. Wahnón, Phys. Rev. B 79, 165203 (2009).

[10]J. Olea, M. Toledano-Luque, D. Pastor, G. González-Díaz, I. Mártil, J. Appl. Phys. 104, 016105 (2008).

[11]E. Antolín, A. Martí, J. Olea, D. Pastor, G. González-Díaz, I. Mártil, A. Luque, Appl. Phys. Lett. 94 (2009).

[12]J. Olea, G. González-Díaz, D. Pastor, I. Mártil, J. of Phys D: Appl. Phys. 42, 085110 (2009).

[13]G. González-Díaz, J. Olea, I. Mártil, D. Pastor, A. Martí, E. Antolín, A. Luque, Sol. Energy Mater. Sol. Cells 93, 1668 (2009).

[14]K. Sánchez, I. Aguilera, P. Palacios, P. Wahnón, submitted to Phys. Rev. B (2010).

[15]M. Tabbal, T. Kim, J. M. Warrender, M. J. Aziz, B. L. Cardozo, R. S. Goldman, J. Vac. Sci. Technol. B 25, 1847 (2007).

[16]M. Tabbal, T. Kim, D. N. Wolf, B. Shin, M. J. Aziz, Appl. Phys. A 98, 589 (2010).

[17]P. Hohenberg, W. Kohn, Phys. Rev. 136, B864 (1964).

[18]W. Kohn, L. J. Sham, Phys. Rev. 140, A1133 (1965).

[19]J. P. Perdew, J. A. Chevary, S. H. Vosko, K. A. Jackson, M. R. Pederson, D. J. Singh, C. Fiolhais, Phys. Rev. B 46, 6671 (1992).

[20] G. Kresse, J. Hafner, Phys. Rev. B 47, 558 (1993).

[21]G. Kresse, J. Furthmüller, Phys. Rev. B 54, 11169 (1996).

[22] G. Kresse, D. Joubert, Phys. Rev. B 59, 1758 (1999).

[23]M. Gajdos, K. Hummer, G. Kresse, J. Furthmüller, and F. Bechstedt, Phys. Rev. B 73, 045112 (2006).

[24]J. Furthmüller, computer code OPTICS, http://www.freeware.vasp.de/VASP/optics/ 